\title{
APPORT DU LIDAR DANS LE GEOREFERENCEMENT D'IMAGES HYPERSPECTRALES EN VUE D'UN COUPLAGE LIDAR/HYPERSPECTRAL
}

\author{
Antoine $\mathrm{Ba}^{1}$, Patrick Launeau ${ }^{1}$, Marc Robin $^{2}$, Saïd Moussaoui ${ }^{3}$, Cyril Michon ${ }^{4}$, Manuel Giraud ${ }^{1}$, Erwan Le Menn ${ }^{1}$ \\ 1 : Laboratoire de Planétologie et Géodynamique de Nantes (LPG-N), Nantes, France \\ antoine.ba@univ-nantes.fr \\ 2 : Littoral - Environnement - Télédétection - Géomatique Nantes (LETG Nantes), Nantes, France. \\ $3:$ Institut de recherche en Communications et Cybernétiques de Nantes (IRCCyN), Nantes, France. \\ 4 : FIT - Conseil, Nantes, France.
}

\begin{abstract}
Résumé
Cette contribution présente deux méthodes de géoréférencement d'images hyperspectrales en se basant sur un jeu de données LiDAR acquises simultanément lors d'une campagne aéroportée en environnement côtier. La première méthode permet de géoréférencer des images VNIR (Visible Near Infra Red) en s'appuyant sur un calcul d'offsets d'angles d'attitude de l'avion basé sur des points de contrôle appariés à un Modèle Numérique d'Élévation (MNE). La seconde méthode a été mise en place dans le but de créer et géoréférencer des images VIR (Visible Infra Red) en fusionnant des images VNIR et SWIR (Short Wave Infra Red). La superposition avec le LiDAR se fait, dans ce cas, par transformation polynomiale de l'image hyperspectrale par saisie de points de contrôle, après géoréférencement. Ces deux méthodes montrent des résultats satisfaisant au vu des échelles d'observation et d'utilisation des données.
\end{abstract}

Mots-clés : Hyperspectral, LiDAR, géoréférencement, offsets, superposition.

\begin{abstract}
This contribution presents two georeferencing methods for hyperspectral images based on a LiDAR data set simultaneously obtained over a coastal environment. The first method allows to georeference VNIR (Visible Near Infra Red) images by computing the attitude angles offsets of the aircraft using Ground Control Point paired to a Digital Elevation Model (DEM). The second method has been established to create and georeference VIR (Visible Infra Red) images by merging VNIR and SWIR (Short Wave Infra Red) images. In the latter case, the superposition with LiDAR data is done by polynomial transformation of the hyperspectral image following a georeferencing step. Both methods have shown satisfactory results regarding the observation and data use scales.
\end{abstract}

Keywords : Hyperspectral, LiDAR, georeferencing, offsets, superposition.

\section{Introduction}

La télédétection, spatiale ou aéroportée, constitue un puissant outil dans le suivi et la gestion de l'environnement. En effet, cette technique permet de couvrir de larges scènes avec une certaines fréquence temporelle. Afin de localiser des objets appartenant à une scène et issus de données multisources ou multitemporelles, il est nécessaire de les positionner dans l'espace. Le géoréférencement, ou rectification des données par rapport à un système de coordonnées local, est une étape critique dans la phase de pré-traitement de données (Müller, 2002). Dans le cas d'images acquises par des capteurs pushbroom aéroportés, le géoréférencement est généralement réalisé après les corrections atmosphériques des données (Cariou and Chehdi, 2008) afin de conserver leurs propriétés radiométriques.
Le couplage entre images hyperspectrales (HSI) et levé topographique LiDAR (Light Detection and Ranging) aéroportés permet d'obtenir des informations sur la nature et la forme des objets présents sur la scène (Brook et al., 2010). Les échelles d'observations de ces objets vont du centimètre à la dizaine de mètre, nécessitant un géoréférencement le plus précis possible. Des travaux ont été réalisés sur le couplage LiDAR/hyperspectral en environnement côtier avec notamment Elaksher (2008) qui a utilisé ce principe afin de cartographier l'occupation du sol et le trait de côte, ou encore Deronde et al. (2004) pour un suivi de la dynamique sédimentaire du littoral belge. La superposition des données LiDAR et hyperspectrales est une phase clé de ce couplage. Celle-ci peut être réalisée par saisie de point de contrôle ou encore par reconnaissance de lignes communes aux images générées par les deux types de capteurs (Avbelj et al., 2013). 
Le but de ce travail de recherche est de présenter une méthode de géoréférencement d'images hyperspectrales à hautes résolutions à partir de données LiDAR en environnement côtier. Le jeu de données LiDAR et HSI présenté ici a été acquis par survol aérien de la façade atlantique de l'lle de Noirmoutier (Vendée, France).

\section{Méthodologie}

\subsection{Zone d'étude}

La zone étudiée se situe à au Nord-Ouest de l'lle de Noirmoutier (Vendée, France), entre la Pointe de l'Herbaudière et la Pointe du Devin (Figure 1). Cette zone présente une plage ouverte d'environ $4 \mathrm{~km}$ recoupée d'ouvrages de défense côtière et bordée d'un cordon dunaire s'élevant de 3 à 4 mètres d'altitude. Ce massif dunaire appelé cordon dunaire de Luzéronde a une largeur variant de $400 \mathrm{~m}$ au Nord à $60 \mathrm{~m}$ au Sud et protège naturellement la zone de marais rétro-littorale (Le Cornec et al., 2008). En termes d'hydrodynamique côtière, cette zone présente une marée à régime macrotidal $(6 \mathrm{~m}$ en vives-eaux) et semi-diurne $(T \sim 12 \mathrm{~h})$ ainsi que qu'une hauteur significative de vague d'environ 1,5m (Dehouck et al., 2013). La dérive littorale opérant sur la zone est globalement Nord-Sud.

\subsection{Matériel aéroporté}

Le jeu de données utilisé dans cette étude a été acquis lors d'une unique campagne aéroportée couvrant une grande partie du littoral vendéen en septembre 2013. II s'agit d'une part d'un ensemble d'images $\mathrm{HSI}$ et d'autre part de nuages de points 3D LiDAR acquis simultanément.

Une image hyperspectrale consiste en un cube représentant une scène observée dont les dimensions $\mathrm{x}$ et $\mathrm{y}$ correspondent à la longitude et la latitude et la dimension $z$ à l'information spectrale. Le nuage de points $3 D$ issu du LiDAR représente des données en longitude $(\mathrm{x})$, latitude $(\mathrm{y})$, élévation (z) ou intensité (i) du retour d'onde du laser.

Les images $\mathrm{HSI}$ ont été acquises grâce à deux capteurs hyperspectraux aux gammes spectrales différentes. Le premier capteur est une caméra HySpex VNIR-1600 (VNIR - Visible and Near InfraRed) dont la gamme spectrale s'étend de 400 à $1000 \mathrm{~nm}$ avec une résolution spectrale de $4.5 \mathrm{~nm}$. Ce capteur pushbroom dont le champ de vue (FOV) est de $17^{\circ}$ produit des images de 1600 pixels across-track. Le second capteur hyperspectral est une caméra HySpex SWIR-320m-e (SWIR - Short Wave Infra Red) dont la gamme spectrale s'étend de 1000 à $2500 \mathrm{~nm}$ avec une résolution spectrale de $6 \mathrm{~nm}$. Ce capteur est également un pushbroom dont le champ de vue de $13,5^{\circ}$ permet de produire des images de 320 pixels across-track. En raison d'un problème technique inattendu lors de la campagne, le système de positionnement ainsi que l'enregistreur inertiel de l'avion (IMU) n'était pas directement connecté aux capteurs hyperspectraux. Les images produites par ces capteurs, nongéoréférencées, seront appelées images brutes et contiennent une information en radiance $\left(\mathrm{W} \cdot \mathrm{m}^{-2} \cdot \mathrm{sr}^{-1}\right)$ (figure 2.a). L'image fusionnée VNIR-SWIR sera notée VIR (Visible Infra Red), couvrant une gamme spectrale de 400 à $2500 \mathrm{~nm}$.

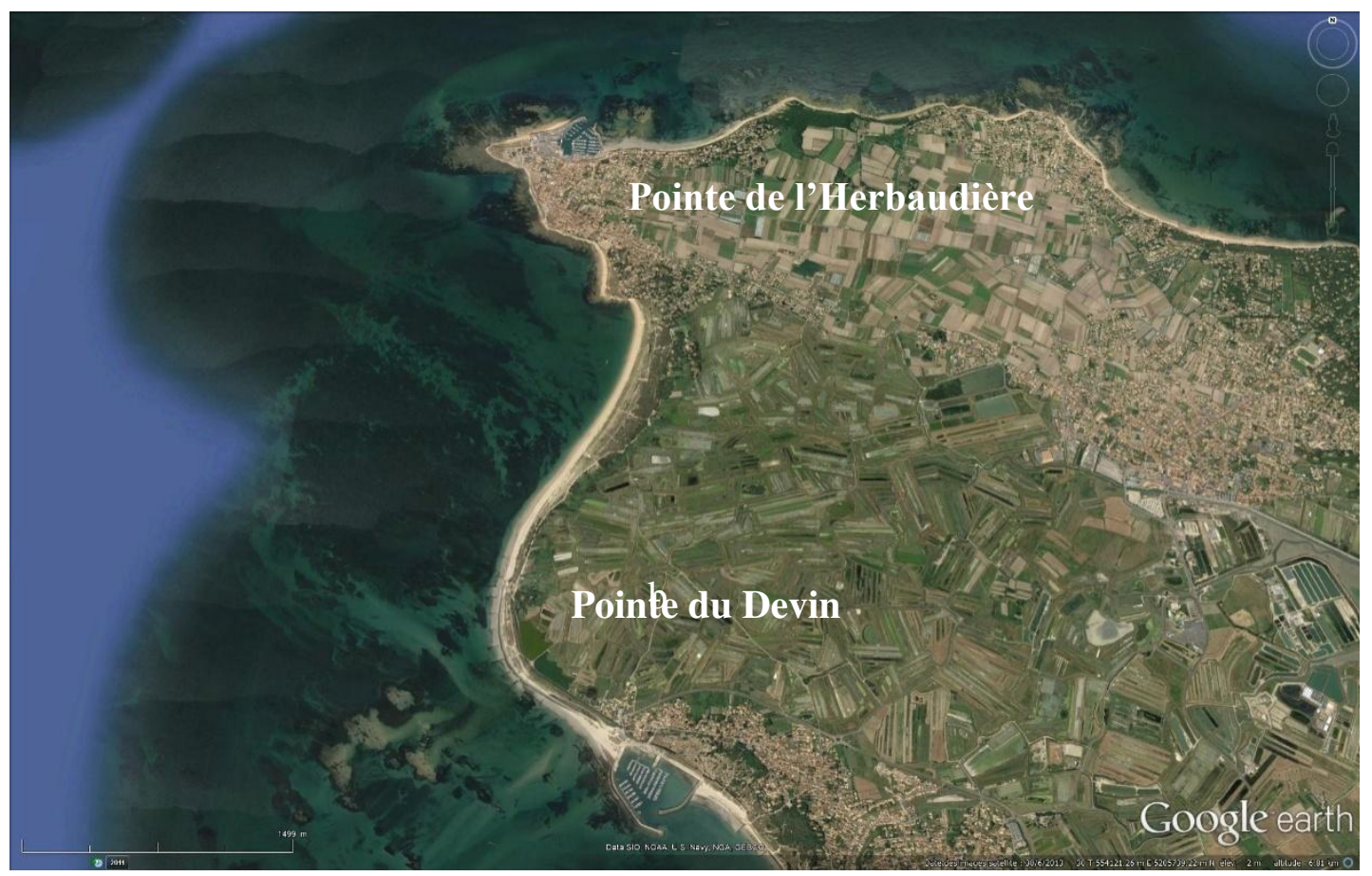

Figure 1 : Zone d'étude, entre la pointe de l'Herbaudière et la Pointe du Devin, lle de Noirmoutier, Vendée, France (source : image Google Earth). 

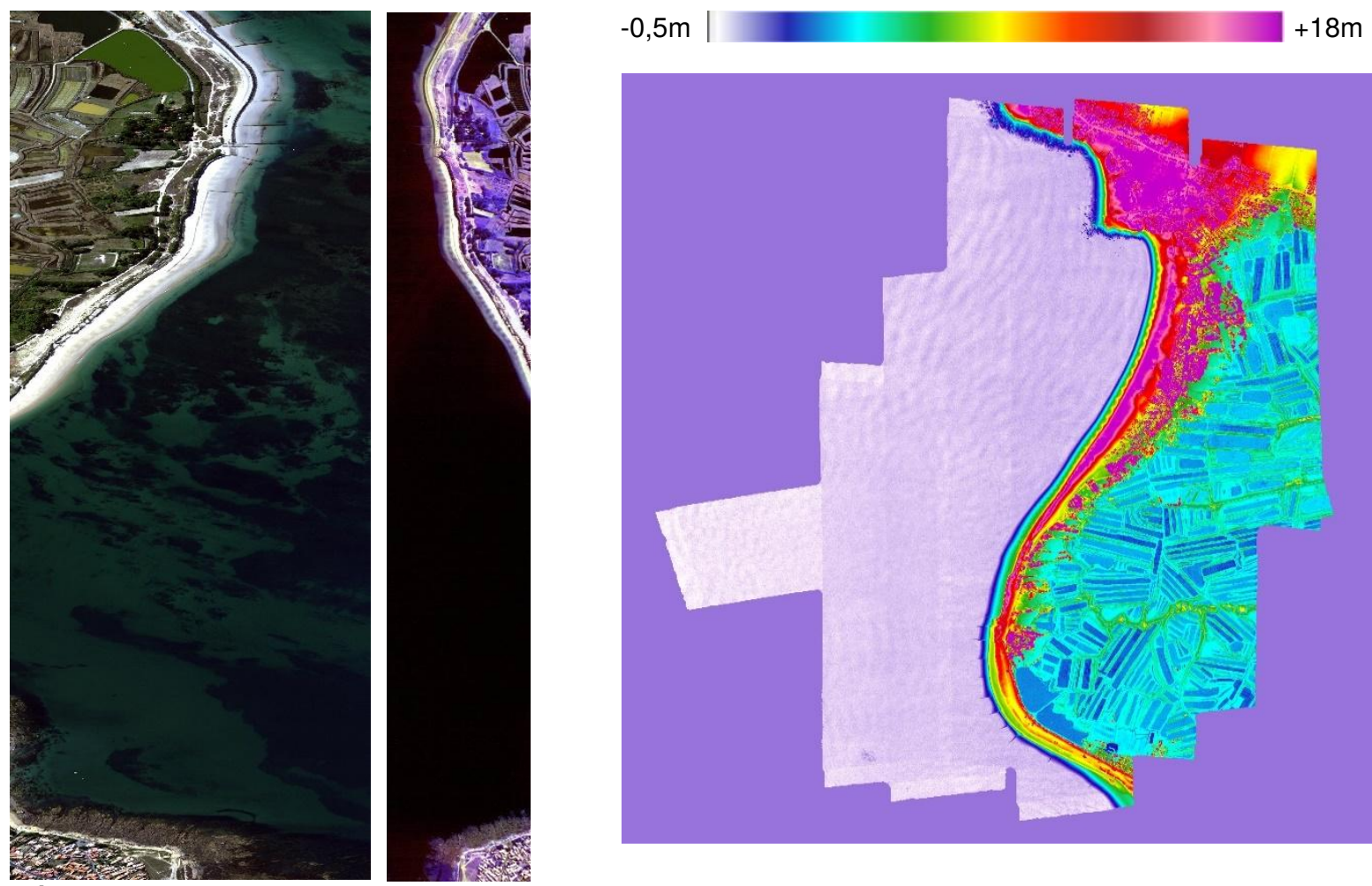

Figure 2 : Couple d'images hyperspectrales brutes VNIR et SWIR (a) en vraies couleurs et Modèle Numérique d'Elévation généré depuis le nuage de points 3D LiDAR (b) à l'ouest de l'lle de Noirmoutier (Vendée, France).

Le LiDAR utilisé lors de cette campagne est un Leica ALS-70 HP, avec une fréquence d'acquisition de $500 \mathrm{kHz}$. La position du LiDAR et les temps d'acquisition pour des points perpendiculaires à la direction de vol sont mesurés par un Global Positioning System (GPS) et une centrale inertielle (Inertial Measurement Unit - IMU) et sont replacés dans le système de référence WGS-84. La longueur d'onde du LiDAR est de $1064 \mathrm{~nm}$, permettant ainsi de récupérer la topographie de la surface survolée. II s'agit d'un LiDAR multipulse enregistrant un seul retour d'onde si la totalité du laser est réfléchie. La densité du nuage de points LiDAR est de 6 points / $\mathrm{m}$ suite à la sélection d'une ouverture de $13^{\circ}$ à $2500 m$ d'altitude de vol pour couvrir au mieux le champ de l'image SWIR la plus étroite. Afin d'exploiter les données LiDAR à la même échelle que les images VNIR et SWIR, celles-ci ont été réparties en dalles représentant les mêmes scènes que les images $\mathrm{HSI}$, à des résolutions similaires, c'est-à-dire $0,5 \mathrm{~m}$ (la taille du pixel du VNIR étant de $0,5 \mathrm{~m}$ ) et $1,8 \mathrm{~m}$ (taille du pixel du VIR étant de $1,8 \mathrm{~m})$.

Ces trois capteurs ont été placés côte à côte dans le vecteur (figure 3.a), un BN2T Islander volant à une altitude de $2500 \mathrm{~m}$ (figure 3.b). Les détecteurs des caméras sont alignés sur une même ligne en avant du lidar. Faute de connexion directe, le bras de levier entre caméras et centre de gravité du lidar et de sa centrale inertielle devra être déterminé a posteriori. La trajectoire du vecteur a été acquise lors de la campagne à l'aide du GPS et de l'IMU associés au LiDAR et a été stockée dans des fichiers dit de trajectographie contenant les données de longitude $\left({ }^{\circ}\right)$, latitude $\left({ }^{\circ}\right)$, altitude $(\mathrm{m})$, roulis $\left({ }^{\circ}\right)$, tangage $\left({ }^{\circ}\right)$, lacet $\left(^{\circ}\right)$ et temps (s). La période d'acquisition de ces données est de 0,0140s.

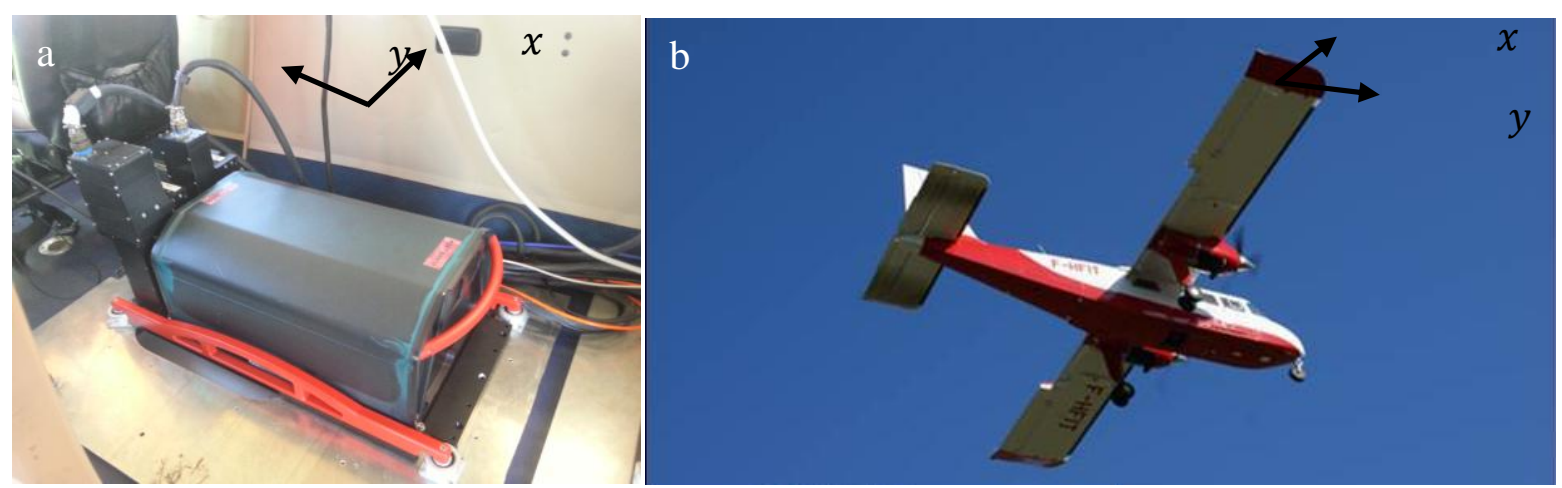

Figure 3 : Montage des capteurs LiDAR et hyperspectraux (a) dans le vecteur BN2T Islander (b). 


\subsection{Géoréférencement des données hyperspectrales à partir des données LiDAR}

Le géoréférencement des données hyperspectrales se fait en deux temps : Une première étape dans le logiciel PARGE (ReSe) qui consiste en la création d'un fichier Input Geometry (IGM) qui permettra, dans la seconde étape sous ENVI (Exelis), de reconstruire l'image géoréférencée.

\subsubsection{Création du fichier Input Geometry}

La création du fichier Input Geometry (IGM) se fait sous PARGE (PARametric GEocoding - ReSe), un logiciel d'orthorectification et de géocodage pour données issues de capteurs optiques aéroportés.

\subsection{1. a. Données en entrées du géocodage}

La première étape est le rééchantillonnage de la trajectographie complète de l'avion afin d'obtenir un fichier de trajectographie propre à l'image brute. Ce rééchantillonnage se fait à l'aide du logiciel HySpex NAV, logiciel associé aux caméras hyperspectrales permettant la segmentation de la trajectographie globale de l'avion en un sous-fichier apparié à l'image brute en se basant sur le temps d'acquisition de la première ligne de pixels de l'image et sur le nombre total de ligne de l'image. Les capteurs HSI n'ayant pas de connexion active au GPS, ce «top » d'acquisition de l'image est obtenu a posteriori par comparaison de la première ligne de l'image $\mathrm{HSI}$ brute avec la ligne de scan du LiDAR correspondante, celle-ci ayant des points associés à des temps d'acquisition. Le fichier de trajectographie en sortie est un fichier contenant le même nombre de ligne que l'image (fichier de trajectographie NAV).

L'étape suivante est la création du fichier IGM. Pour ce faire, le logiciel PARGE a été utilisé, avec comme entrées l'image brute, le fichier de trajectographie NAV, le fichier de définition du capteur, le Modèle Numérique d'Élévation (MNE) haute résolution issu du LiDAR ainsi que la projection et le datum de géoréférencement des images (ici UTM 30 Nord WGS-84).

\subsection{1. b. Algorithmes de géocodage}

Les équations utilisées dans le logiciel PARGE sont issues des travaux de Derenyi et Konecny (1966) et améliorées par Konecny (1972; 1976). La chaîne de traitements présentée ici est décrite dans le manuel du logiciel PARGE (Schläpfer, 2011).

La phase de calcul commence avec le vecteur de capteur $\overrightarrow{L_{0}^{\prime}}$ (Figure 4 ) qui est calculé en se basant sur les coordonnées $x$ (pixel) et $y$ (ligne) du système de coordonnée du capteur.

Le système de coordonnée du capteur est ensuite converti à l'aide des angles d'attitude roulis, tangage et lacet en un système de coordonnées défini en entrée du traitement :

$\overrightarrow{L_{0}}=$ R.P.H. $\overrightarrow{L_{0}^{\prime}}$

$$
\begin{aligned}
& \text { Soit } \\
& {\left[\begin{array}{l}
L_{0, x} \\
L_{0, y} \\
L_{0, z}
\end{array}\right]=} \\
& {\left[\begin{array}{ccc}
\cos \phi & 0 & -\sin \phi \\
0 & 1 & 0 \\
\sin \phi & 0 & \cos \phi
\end{array}\right]\left[\begin{array}{ccc}
1 & 0 & 0 \\
0 & \cos \theta & \sin \theta \\
0 & -\sin \theta & \cos \theta
\end{array}\right]\left[\begin{array}{ccc}
\cos \psi & \sin \psi & 0 \\
-\sin \psi & \cos \psi & 0 \\
0 & 0 & 1
\end{array}\right]\left[\begin{array}{l}
L_{0, x^{\prime}} \\
L_{0, y^{\prime}} \\
L_{0, z^{\prime}}
\end{array}\right]}
\end{aligned}
$$

Où $\mathrm{R}, \mathrm{P}$ et $\mathrm{H}$ correspondent aux matrices associées au roulis, tangage et lacet. Les angles d'attitude de l'avion sont intégrés comme des angles de rotation avec $\phi$ pour le roulis, $\theta$ pour le tangage et $\psi$ pour le lacet.

Il est ensuite possible de calculer la longueur du vecteur du capteur en utilisant l'altitude $\mathrm{h}$ de l'avion afin d'obtenir le vrai vecteur $\overrightarrow{L_{t}}$ :

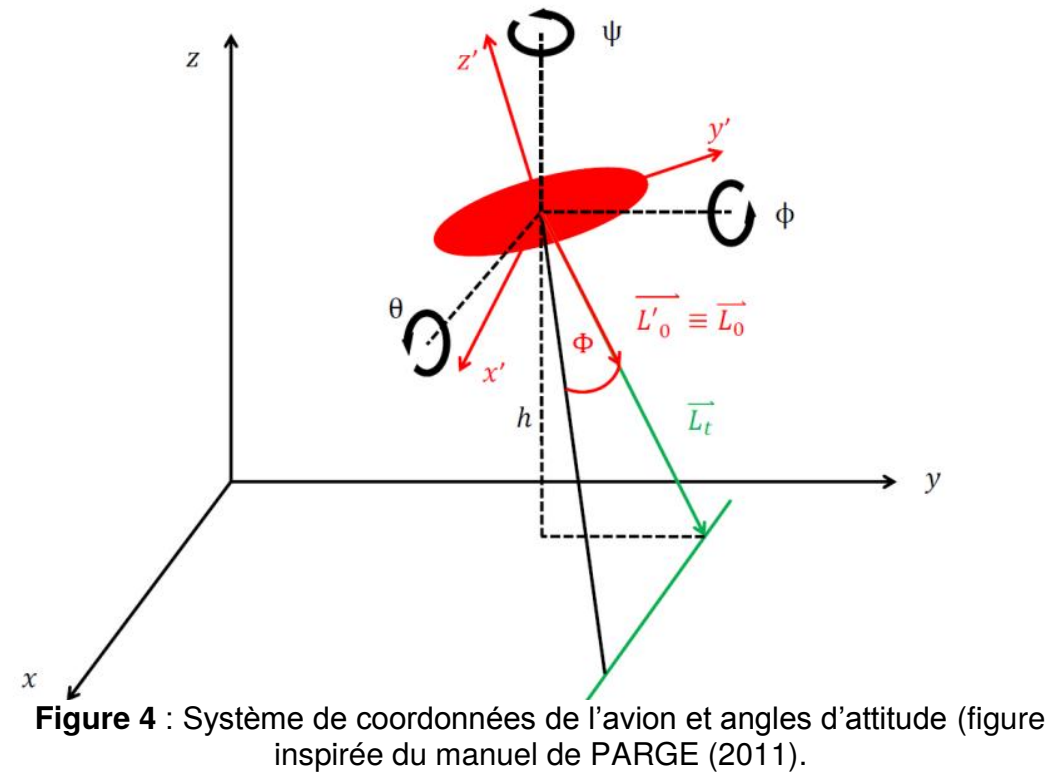




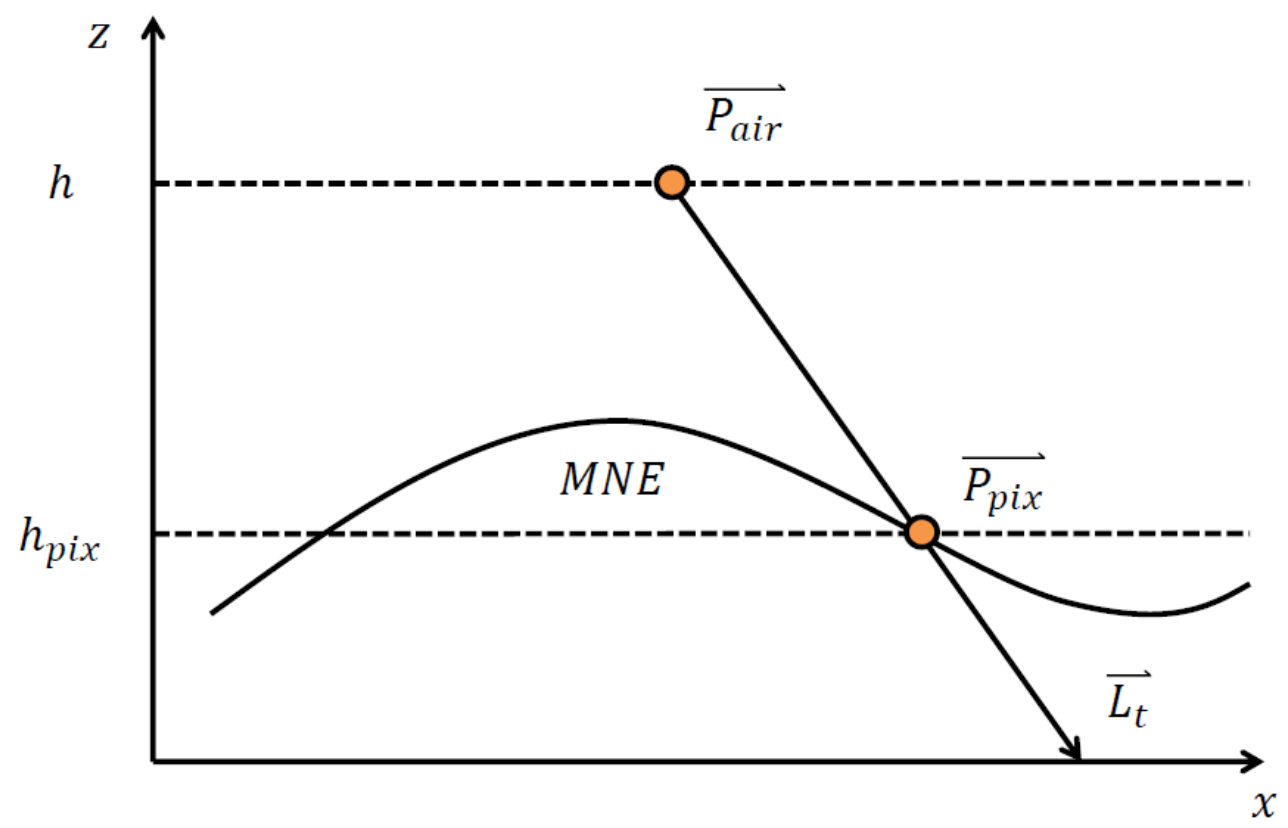

Figure 5 : Extraction de la position de la ligne de pixel dans le référentiel associé aux images HSI (figure inspirée du manuel de PARGE (2011)).

$\overrightarrow{L_{t}}=\frac{-h}{L_{0, z}} \overrightarrow{L_{0}}$

Le géocodage de l'image doit dans l'idéal prendre en compte l'élévation du terrain. Le logiciel PARGE utilise une procédure de lancer de rayon afin de retrouver la position de la ligne de pixels visée à partir de la position de l'avion $\overrightarrow{P_{a r r}}(t)$ (Figure 5). La position de la ligne de pixels $\overrightarrow{P_{p l x}}(t)$ correspond à l'intersection du vecteur $\overrightarrow{L_{t}}$ avec le MNE (Figure 5).

\subsection{1. c. Intégration de points de contrôle}

Après application de cette phase de géoréférencement, quelques distorsions restent visibles entre les images dérivées du LiDAR et les images HSI géoréférencées. Ces distorsions sont causées par un manque de précision i) du temps GPS en raison du pointage visuel a postériori du " top » départ de l'acquisition des images mais aussi ii) des angles d'attitude de l'avion associés au LiDAR en raison de l'impossibilité momentanée de calculer précisément bras de levier et distorsion angulaires entre les caméras et l'ensemble GPS IMU (boresight calibration).

Afin d'obtenir malgré tout des images hyperspectrales le plus proche possible des images LiDAR considérées comme reflétant la réalité, la phase de géocodage est complétée par intégration de points de contrôle. Ces points de contrôle sont saisis et entrés manuellement dans PARGE, appariés entre une image HSI brute et le MNE correspondant. Ils sont principalement des points fixes observés sur la scène, type coin de bâtiments ou arbres isolés. Ils doivent être clairement distinguables sur l'image $\mathrm{HSI}$ et sur le MNE.
L'image en intensité du retour d'onde LiDAR peut constituer une aide supplémentaire à leur définition. Le MNE utilisé est ajusté sur l'ellipsoïde de référence terrestre, c'est-à-dire que les valeurs d'altitude sont de :

$z=z_{I G N}+47 m$

Avec $Z_{I G N}$ l'élévation dans le référentiel de l'Institut National de l'Information Géographique et Forestière (IGN) et $47 \mathrm{~m}$ la hauteur de l'ellipsoïde de référence par rapport au zéro IGN dans la zone survolée. Le MNT utilisé pour les images VNIR a une taille de pixel de $0,5 \mathrm{~m}$.

L'intégration des points de contrôle permet de créer une position estimée de l'avion :

$\overrightarrow{P_{a}^{\prime}}=\overrightarrow{P_{G C P}}-\overrightarrow{L_{t}} \frac{h_{a}-h_{G C P}}{h\left(\overline{L_{t}}\right)}$

Avec $\overrightarrow{P_{a}^{\prime}}$ la position estimée de l'avion, $\overrightarrow{P_{G C P}}$ le vecteur position du point de contrôle et $h_{a}$ et $h_{G C P}$ leurs altitudes absolues respectives. Les offsets des angles d'attitude sont obtenus par différence entre les angles d'attitude estimés et les véritables angles d'attitude de l'avion (issue des données GPS) (Figure 6). Pour chaque angle d'attitude, un unique offset est calculé en moyennant les valeurs des offsets des points de contrôle. Ces offsets serviront à corriger les angles d'attitude de l'avion. Par expérience, la saisie d'une quinzaine de points de contrôle maximum suffit à procéder à l'opération. 


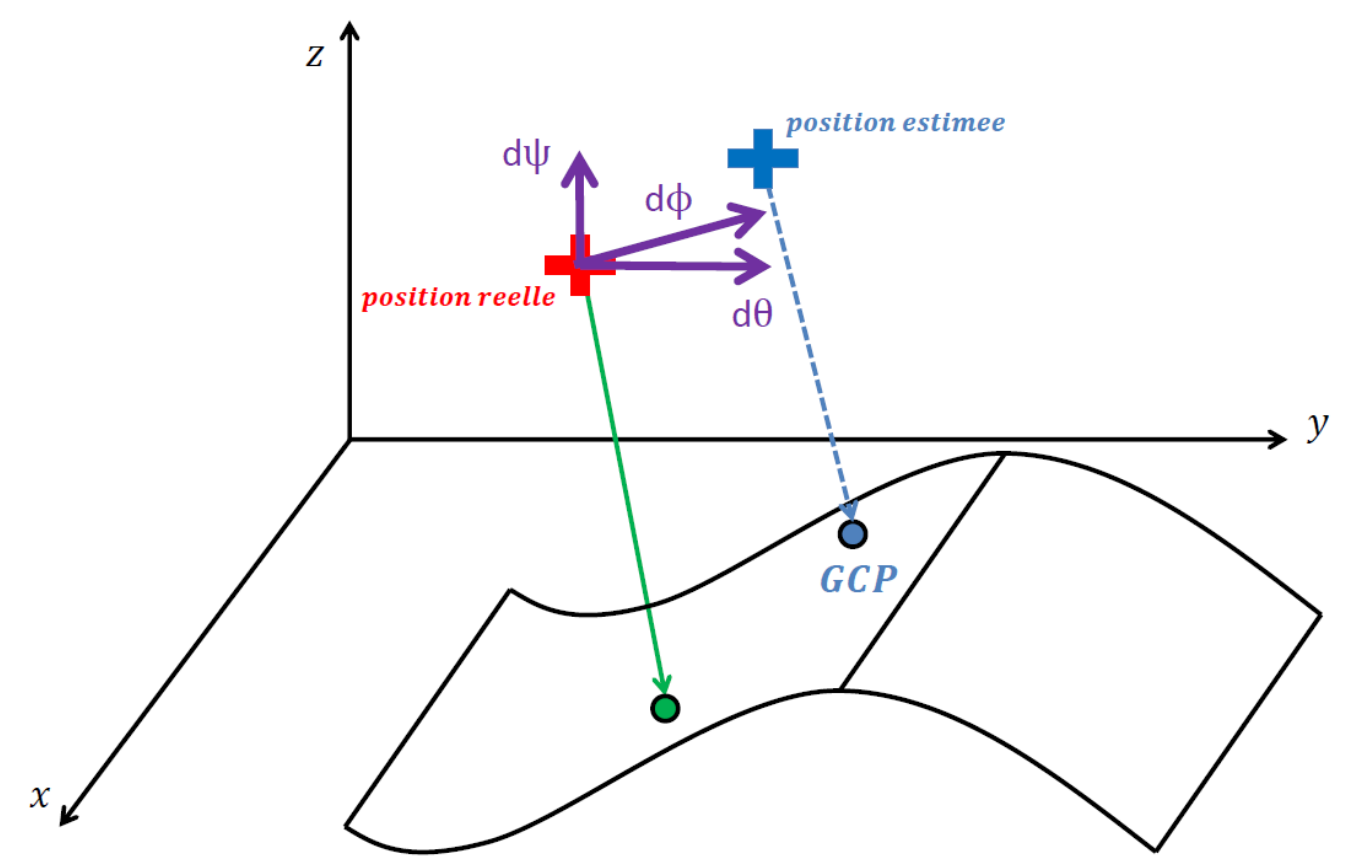

Figure 6 : Calcul des offsets de roulis $(d \phi)$, tangage $(d \theta)$ et lacet de l'avion $(d \psi)$.

Après saisie image à MNT des points de contrôle et calcul des valeurs d'offsets dans PARGE, un fichier d'Input Geometry est créé. Ce fichier représente la position des pixels de l'image brute en longitude et latitude.

Dans le cas de la création du fichier Input Geometry dans le but de superposer des images VNIR et SWIR, l'intégration des points de contrôle n'est pas réalisée. En effet, on observe lors du géoréférencement des images HSI VNIR et SWIR, qu'elles sont simplement translatées l'une par rapport à l'autre. La saisie de point de contrôle modifierait cette translation en une déformation polynomiale qui rendrait plus complexe une future superposition VNIR-SWIR. Dans le but de créer une image VIR, le fichier IGM est créé en se basant sur les valeurs par défaut de la trajectographie et de l'attitude de l'avion. La superposition des images VNIR et SWIR ne peut se faire avant géoréférencement car cette étape nécessite que chaque pixel des deux images ait une position en longitude et latitude.

\subsection{Géoréférencement de l'image}

L'étape de géoréférencement est, dans cette procédure, réalisée dans le logiciel ENVI. Un des outils disponibles dans le logiciel permet de géoréférencer des images grâce à un fichier IGM. Dans notre étude, il a été choisi de géoréférencer les images avec une taille de pixel donnée par défaut puis de rééchantillonner les images à une résolution commune de $0,5 \mathrm{~m}$ pour le VNIR et $1,8 \mathrm{~m}$ pour le VIR, par le biais de la méthode pixel aggregate. Cette méthode permet de recréer un pixel en fusionnant les informations de pixels adjacents.
Les images VIR seront calées sur le LiDAR par une méthode de co-registration d'images basé sur la saisie d'un grand nombre de points de contrôle $(\sim 150)$. Cette méthode permet de trouver la meilleure solution du polynôme de déformation entre l'image $\mathrm{HSI}$ et le LiDAR.

Les images $\mathrm{HSI}$ sont ensuite comparées aux images en intensité issues du LiDAR afin d'évaluer la qualité du géoréférencement. Pour ce faire, une image composite des bandes \#2 et \#10 de l'image $\mathrm{HSI}$ et de la dalle LiDAR en intensité est créée. Une image de la zone surveillée sera présentée dans les résultats. La figure 7 récapitule l'ensemble des procédures décrites précédemment.

\section{Résultats}

\subsection{Géoréférencement d'images VNIR}

La figure 7 montre l'évolution des angles d'attitude de l'avion en fonction du numéro de la ligne de l'image. Les valeurs d'offsets sont de $0,317^{\circ}$ pour le roulis, $0,356^{\circ}$ pour le tangage et $0,247^{\circ}$ pour le lacet. Les valeurs des angles utilisées pour le géoréférencement des images HSI correspondent aux valeurs d'angles par défaut auxquelles sont ajoutés les offsets.

Le géoréférencement des images $\mathrm{HSI}$ est évalué par superposition aux images en intensité LiDAR. $\mathrm{La}$ figure 8.a. représente une image $\mathrm{HSI}$ géoréférencée de la zone d'étude. Sur la figure 8.b, cette même image (Rouge et Vert) est superposée à la dalle LiDAR en intensité correspondante (Bleu). On peut voir sur les zooms (Figures 8.c, 8.d, 8.e) qu'il existe un décalage résiduel HSI/LiDAR. En effet, en termes de pixels, on retrouve une image HSI décalée de 2 pixels vers l'Est et aucun décalage vers le Nord sur la figure 8.c, alors que sur 
la figure 8.d, on retrouve un décalage plus important, de 4 pixels vers l'Ouest et 4 pixels vers le
Sud. La figure 8.e ne présente cependant aucun décalage spatial entre l'image HSI et la dalle LiDAR.

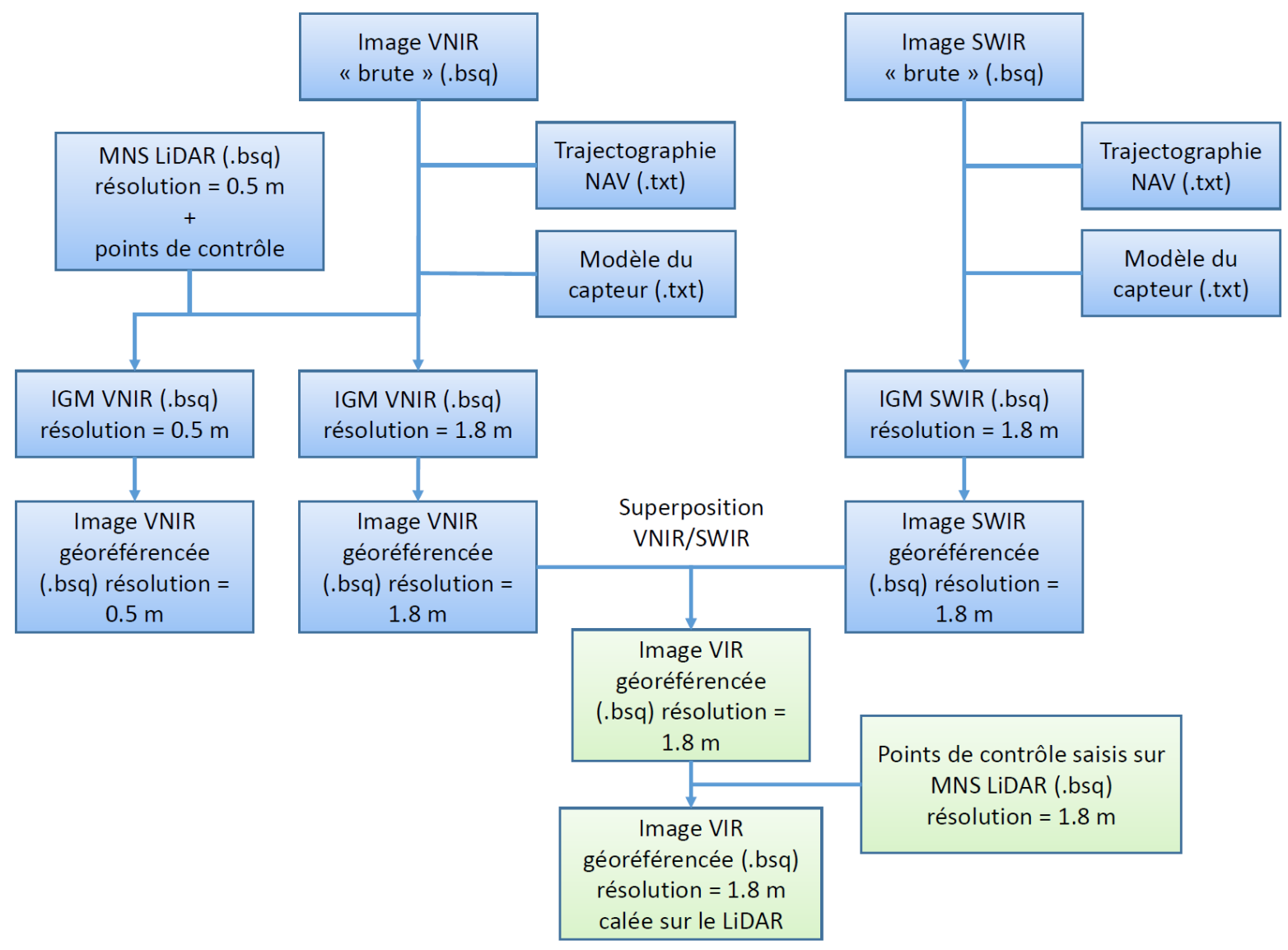

Figure 7 : Diagramme synthétique des procédures de géoréférencement d'images hyperspectrales VNIR et VIR à l'aide d'un MNS issu du LiDAR.
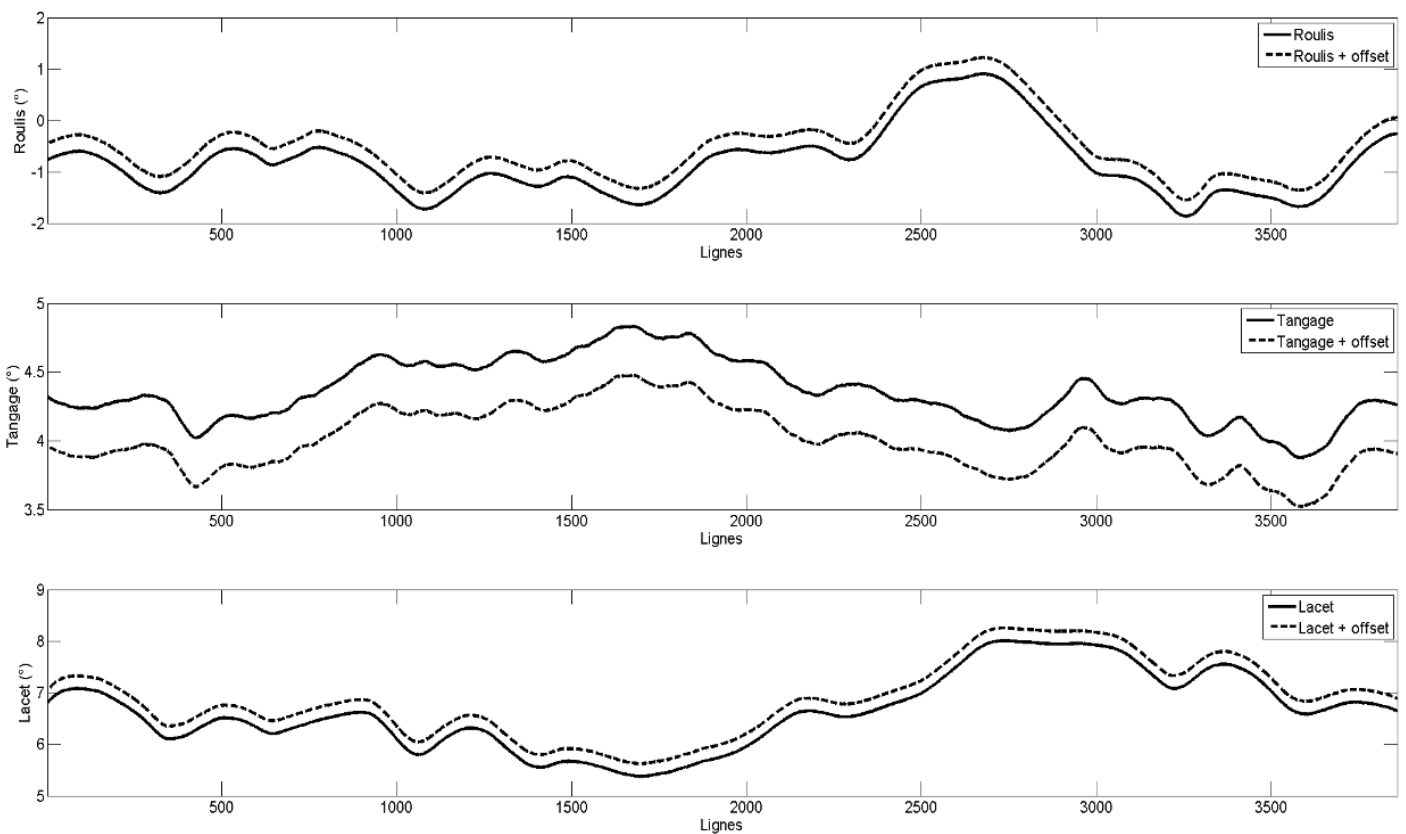

Figure 8 : Evolution du roulis, tangage et lacet associée à celle de leur offset respectif en fonction du numéro de ligne de l'image. 

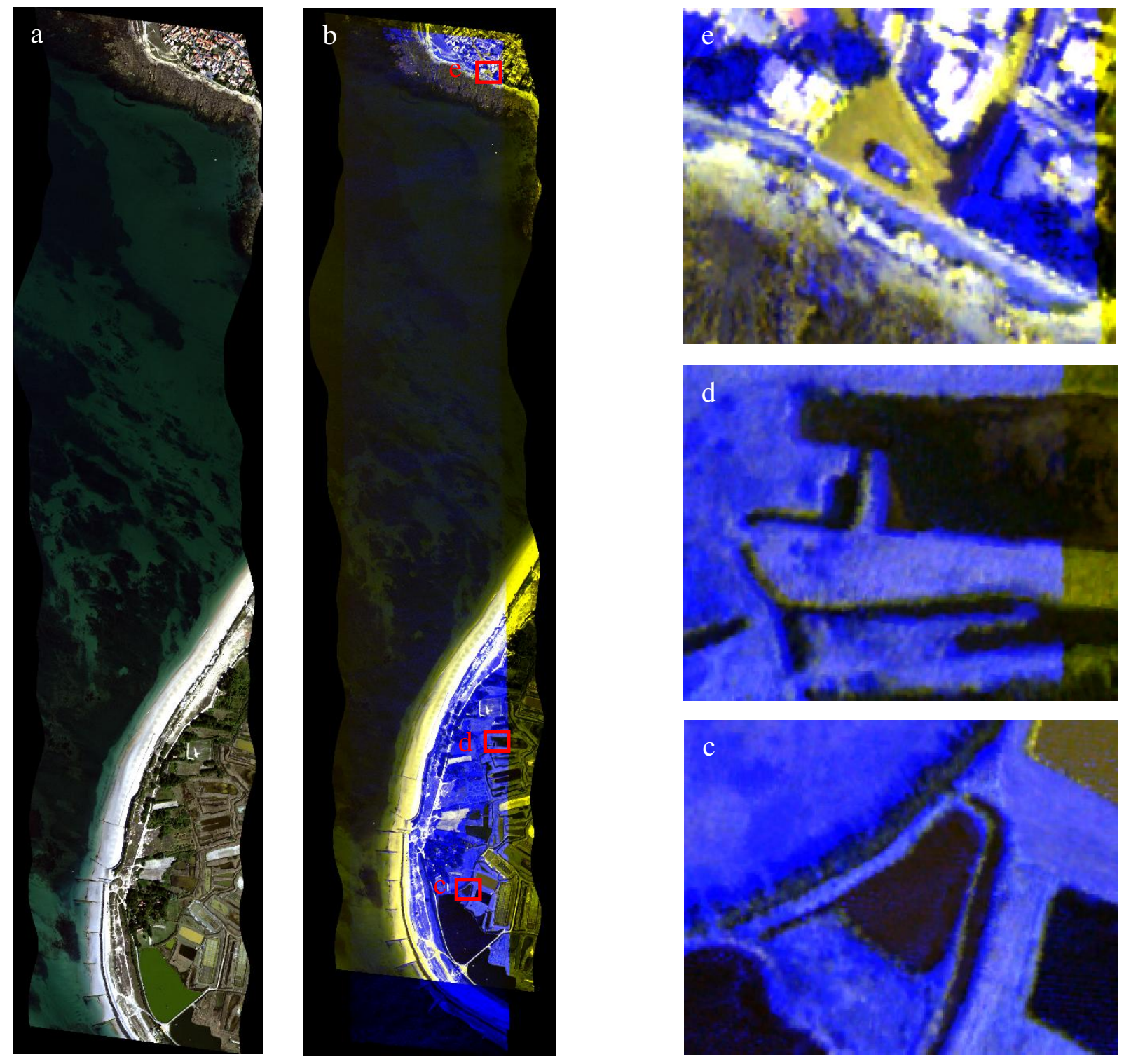

Figure 9 : Image hyperspectrale VNIR (a) de l'ouest de l'lle de Noirmoutier (Vendée, France) et sa superposition au LiDAR (b). (c), (d) et (e) représentent des zooms sur l'image (Rouge : VNIR - 413,74 nm, Vert : VNIR 442,76 nm, Bleu : LiDAR - intensité).

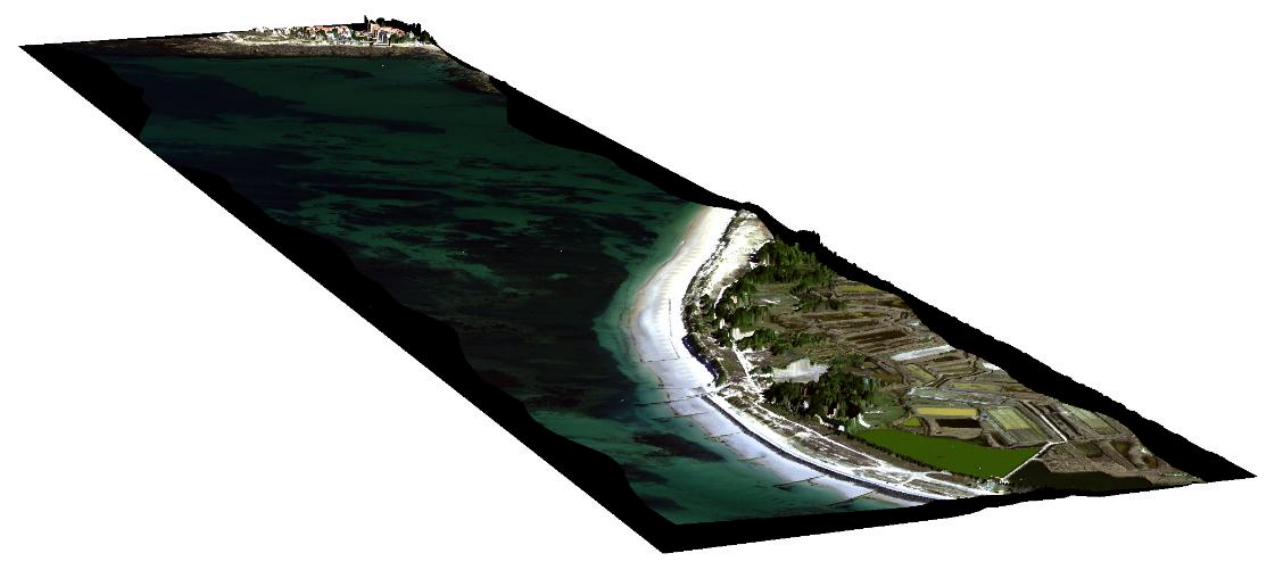

Figure 10 : Image hyperspectrale 3D issue du drapage de l'image HSI sur le MNE issu du LiDAR. Ce type d'image donne accès à une information en $4 \mathrm{D}$, utile dans différents domaines type génie civil et côtier. 
On observe donc sur cette image VNIR des décalages maximaux de 4 pixels et minimaux de 0 pixels en latitude et longitude. Cela représente des variations allant de 0 à $4 \mathrm{~m}$ sur les deux dimensions. II faut noter que les décalages avec le LiDAR ne sont pas fixes d'une ligne à l'autre de l'image.

La figure 9 permet d'appréhender la notion de cartes hyperspectrales 3D. II est également observable sur cette figure le décalage entre le MNE issu du LiDAR et l'image HSI.

\subsection{Géoréférencement d'images VIR}

La création d'images VIR géoréférencées se fait par superposition d'image VNIR et SWIR à la résolution $1,8 \mathrm{~m}$. Les images VNIR et SWIR brutes sont d'abord géoréférencées par Input Geometry sans prendre en compte le décalage avec le LiDAR. Sur la figure 10 , on peut voir que le décalage VNIRSWIR est constant avec des valeurs de 4 pixels $(7,2 \mathrm{~m})$ vers l'Est et 0 pixels en latitude grâce au choix original de l'alignement des caméras entre elles réalisée pour le LPG-N (figure 3). Ce décalage est corrigé par pure translation de l'image

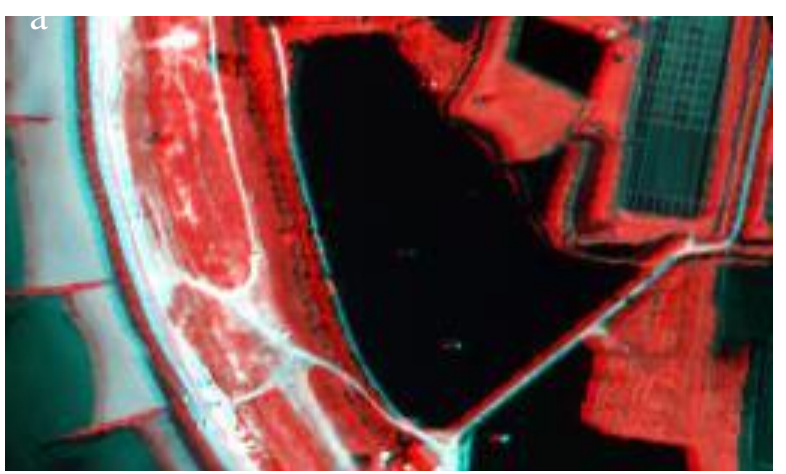

Figure 11 : Superposition des images VNIR et SWIR avant (a) et après (b) correction du décalage (pure translation) (Rouge : SWIR - 1020,01 nm, Vert : VNIR - 442,76 nm, Bleu : VNIR - 413,74 nm).
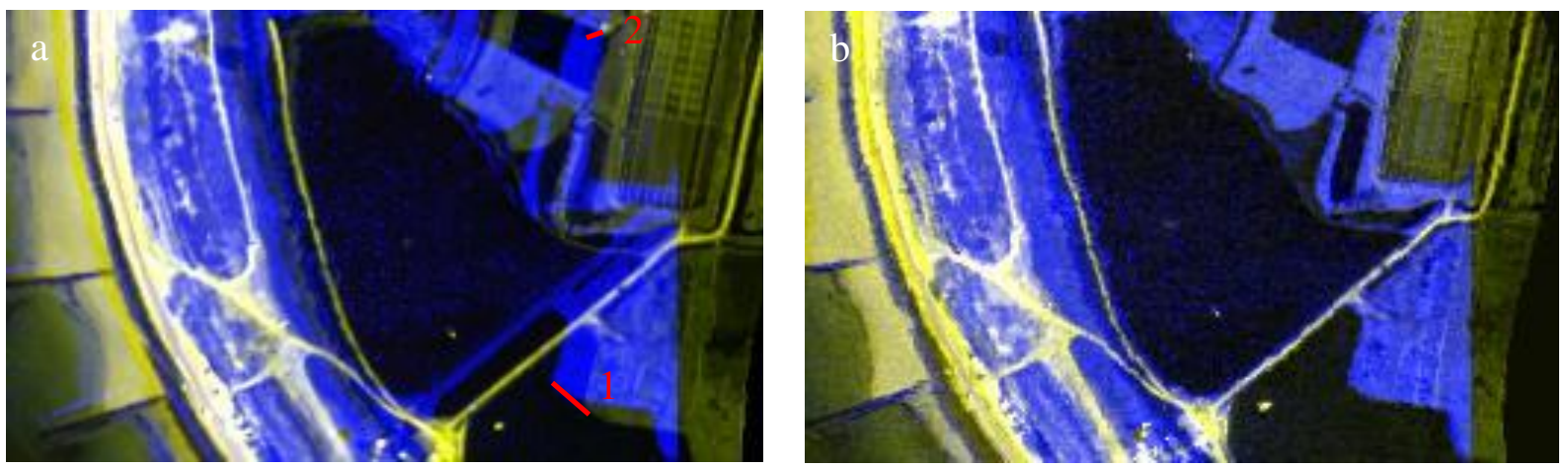

Figure 12 : Superposition de l'image hyperspectrale VIR et de la dalle LiDAR correspondante avant (a) et après correction polynomiale par points de contrôle (b) (Rouge : VNIR - 413,74 nm, Vert : VNIR - 442,6 nm, Bleu : LiDAR - intensité)

SWIR par rapport à la VNIR, ce qui permet d'obtenir des images VIR spatialement cohérente. Les images sont ensuite fusionnées. Cependant, les offsets des angles d'attitude de l'avion n'ayant pas été pris en compte (toujours pour les mêmes raisons techniques) dans la création des Input Geometries, un fort décalage existe entre VIR et LiDAR (figure 11.a). A titre indicatif, le segment 1 (figure 11.a) représente un décalage de 11 pixels $(19,8 \mathrm{~m})$ en longitude et 8 pixels $(14,4 \mathrm{~m})$ en latitude alors que le segment 2 montre des valeurs de 6 pixels $(10,8 \mathrm{~m})$ en longitude et 1 pixel $(1,8 \mathrm{~m})$ en latitude. La saisie d'une centaine de points de contrôle permet de récupérer une image superposée au LiDAR au pixel près mais cette méthode est très gourmande en temps.

\section{Discussion}

\subsection{Qualité du géoréférencement des images VNIR}

La précision des résultats obtenus dans le calage VNIR/LiDAR est satisfaisante compte tenu du manque d'informations associées aux capteurs hyperspectraux. En effet, en utilisant les données de l'IMU et du GPS du LiDAR, on associe un temps, une position et une attitude sans connexion directe

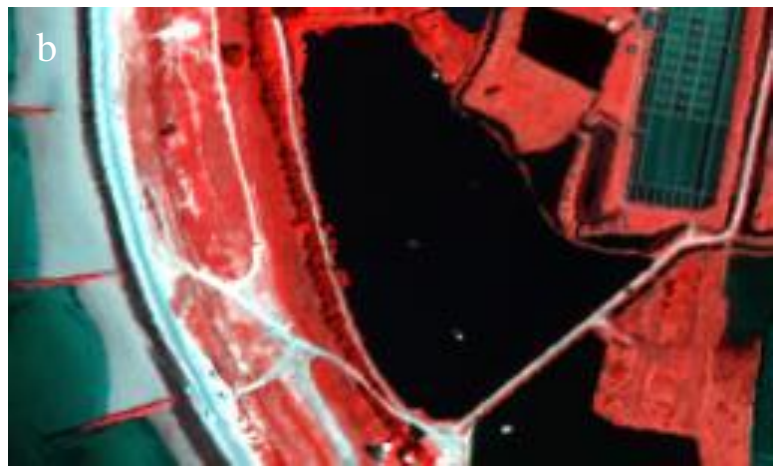


angles d'attitudes de l'avion varient d'une ligne à l'autre (Figure 7) en raison d'un manque de précision dans la détermination du top image. II est donc impossible d'obtenir un décalage unique valable sur toute l'image. Afin d'augmenter la précision de ce géoréférencement, il faudrait:

Rétablir l'acquisition simultanée du temps GPS (et non a posteriori) pour réaliser une calibration parfaite des angles de visée et des différents bras de levier entre caméras et GPS/IMU du LiDAR (boresight calibration);

- Parvenir à minimiser les offsets dans PARGE en saisissant plus de point de contrôle ;

- Ajouter une nouvelle étape à la procédure en s'inspirant du calage VIR-LiDAR et en saisissant de nouveaux points de contrôle après géoréférencement ;

Le saisie de points de contrôle est l'étape critique du géoréférencement d'images VNIR et représente également l'étape la plus couteuse en temps humain. Afin de saisir une quinzaine de points de contrôle de créer un fichier IGM, l'utilisateur doit compter $30 \mathrm{mn}$ de temps de travail.

\subsection{Qualité du géoréférencement des images VIR}

La procédure mise en place pour le géoréférencement d'images VIR est relativement longue et lourde. En effet, même si la saisie de points de contrôle est supprimée dans la phase de création du fichier IGM, celle-ci est nécessaire après géoréférencement. Le nombre de points de contrôle entré dans cette procédure est très largement supérieur à la dizaine de points nécessaire dans le géoréférencement des images VIR. Cependant on retrouve, après co-registration, des images VIR et LiDAR avec un décalage résiduel faible, donc un géoréférencement de qualité. Avec cette procédure, il ne sera nullement nécessaire de retravailler les données en post-traitement. La création de deux fichiers IGM et le grand nombre de points de contrôle saisi pour le géoréférencement d'images VIR accroissent le coût humain et machine à environ $45 \mathrm{mn}$ à $1 \mathrm{~h}$ pour un couple d'image.

\subsection{Notions d'échelles d'observations}

L'objet d'étude ici est de fournir une procédure qui permet d'exploiter des images hyperspectrales en couplage avec des données LiDAR en environnement côtier. Les échelles d'observation des éléments des scènes allant du mètre (végétation dunaire) à la dizaine-centaine de mètre (morphologie de la plage), il est nécessaire d'obtenir la meilleure superposition des données possible. Dans notre étude, les décalages résiduels VNIR/LiDAR pourront être restreints par comparaison de l'information spectrale et de l'élévation ou de l'intensité du retour d'onde LiDAR d'un objet observé. Cette future étape relève du post-traitement des données en vue d'une application thématique. Cependant, dans d'autres domaines d'applications, le décalage images HSILiDAR peut ne pas être très contraignant. En effet, l'observation à des échelles allant de la centaine de mètres au kilomètre dans des milieux plus homogènes que l'environnement littoral peut s'affranchir d'un géoréférencement précis comme celui présenté ici.

\section{Conclusion}

Dans cette étude, deux méthodes de géoréférencement a posteriori d'images hyperspectrales VNIR et VIR ont été présentées. La première permet de géoréférencer les images VNIR en s'appuyant sur la création de nouveaux angles d'attitude d'avion à l'aide de points de contrôle. La résultante est une image présentant des décalages spatiaux variables par rapport au LiDAR dont le calage géographique fourni par FIT-Conseil à l'aide de la chaine de traitement de LEICA est optimum. Ces décalages atteignent jusqu'à $2 \mathrm{~m}$ en longitude et latitude. La seconde méthode permet de coupler des images VNIR et SWIR afin d'obtenir une image VIR couvrant la gamme spectrale 400-2500 nm avec une seule co-registration à l'image d'intensité du LiDAR.

Idéalement, les capteurs hyperspectraux devraient être munis d'un GPS afin de synchroniser le début des acquisitions. L'apparition d'incident technique peut être cependant maîtrisée par le fait que l'acquisition des images ait été faite à partir de la même plateforme aéroportée ce qui divise les coûts de mise en œuvre aéroportée par deux (une seule campagne au lieu de deux). Ainsi, avec la résolution des problèmes de connexion entre systèmes, le couplage des données pourrait être fait plus précisément et il y aurait un gain de temps considérable. De plus, cette méthode pourrait être affinée et partiellement automatisée en intégrant un outil de fusion de données automatique basé sur la reconnaissance de formes (Brook et al., 2010) ou sur des descripteurs robustes d'éléments de la scène observée (Montero et al., 2013).

\section{Références}

Avbelj, J., Iwaszczuk, D., Müller, R., Reinartz, P., Stilla, U., 2013. Line-based registration of DSM and hyperspectral images. International Archives of the Photogrammetry, Remote Sensing and Spatial Information Sciences, 60, ISPRS Hannover Workshop, Hannover, Germany.

Brook, A., Ben-Dor, E., Richter, R., 2010. Fusion of hyperspectral images and LiDAR data for civil 
engineering structure monitoring. Proceedings of Hyperspectral 2010 Workshop, Frascati, Italy, 1-5.

Dehouck, A., Kervella, S., Lafon, V., Curti, C., Alexandre, F., Chaumillon, E., Bertin, X., Sottolichio, A., Robin, N., Naulleau, P., Vanroye, C., 2013. The Arcay spit and Lay estuary (Vendee, France): New data to highlight sediment dynamics and support coastal management. Proceedings of Coastal Dynamics 2013, Arcachon, France.

Derenyi, E. E., Konecny, G., 1966. Infrared scan geometry. Photogrammetry Engineering, 32, 773779 .

Deronde, B., Houthuys, R., Sterckx, S., Debruyn, W., Fransaer, D., 2004. Sand dynamics along the belgian coast based on airborne hyperspectral data and LiDAR data. Proceedings of the European Association of Remote Sensing Laboratories, 3.

Elaksher, A., 2008. Fusion of hyperspectral images and LiDAR-based DEMs for coastal mapping. International Archives of the Photogrammetry, Remote Sensing and Spatial Information Sciences, 32, Part B3b, Beijing, China.

Konecny, G., 1972. Geometrical aspects of remote sensing. Invited paper of International Congress of Photogrammetry, Commission IV, Ottawa, Canada, pp.47.
Konecny, G., 1976a. Mathematical models and procedures for the geometric restitution of remote sensing imagery. Invited paper of the XIII International Congress of Photogrammetry, Commission III, Helsinki, Finland, pp. 33.

Konecny, G., 1976b. Mathematische Modelle und Verfahren zur geometrischen Auswertung von Zeillenabtaster-Aufnahmen. Bildmessung u. Luftbildwesen, 44, 188-197.

Le Cornec, E., Fiere, M., Grunnet, N., 2008. Etude de connaissance des phénomènes d'érosion sur le littoral vendéen. Rapport de la Danish Hydraulics Institute France pour la DDE85.

Le Cozannet, G., Lecacheux, S., Delvallee, E., Desramaut, N., Oliveros, C., Pedreros, R., 2011. Teleconnection pattern influence on sea-wave climate in the Bay of Biscay. Journal of Coastal Research, 24, 641-652.

Monteiro, S. T., Nieto, J., Murphy, R., Ramakrishnan, R., Taylor, Z., 2013. Combining strong features for registration of hyperspectral and LiDAR data from field-based platforms. IEEE International Geoscience and Remote Sensing Symposium, Melbourne, Australia.

Schläpfer, D., 2011. PARGE user manuel version 3.1. ReSe, Zurich, Switzerland. 